\title{
Shadowgraphic studies of triazene assisted laser-induced forward transfer of ceramic thin films
}

\author{
K. S. Kaur, ${ }^{1, a)}$ R. Fardel, ${ }^{2,3}$ T. C. May-Smith, ${ }^{1}$ M. Nagel, ${ }^{3}$ D. P. Banks, ${ }^{1}$ C. Grivas, ${ }^{1}$ \\ T. Lippert, ${ }^{2}$ and R. W. Eason ${ }^{1}$ \\ ${ }^{1}$ Optoelectronics Research Centre, University of Southampton, Southampton SO17 1BJ, United Kingdom \\ ${ }^{2}$ General Energy Research Department, Paul Scherrer Institut, 5232 Villigen PSI, Switzerland \\ ${ }^{3}$ Laboratory for Functional Polymers, EMPA-Swiss Federal Laboratories for Materials Testing and \\ Research, Überlandstrasse 129, 8600 Dübendorf, Switzerland
}

(Received 4 December 2008; accepted 20 April 2009; published online 9 June 2009)

\begin{abstract}
The laser-induced forward transfer process of solid ceramic donor materials (gadolinium gallium oxide and ytterbium doped yttrium aluminium oxide) was studied using triazene polymer as a sacrificial layer by means of a time-resolved nanosecond-shadowgraphy technique. The dependence of the ablation dynamics and quality of the ejected donor material on the laser fluence and thickness of the sacrificial and donor layers were investigated and discussed. (ㅇ 2009 American Institute of Physics. [DOI: 10.1063/1.3132822]
\end{abstract}

\section{INTRODUCTION}

The laser-induced forward transfer (LIFT) method has received a great deal of attention in the past two decades as an additive laser direct-write technique due to its simplicity, versatility, and the potential advantages it offers. The basic working principle of LIFT involves the focusing of a laser pulse through a transparent support substrate (the carrier) onto the rear side of a thin film of the material to be LIFTed (the donor). Another substrate (the receiver) onto which the donor material is to be transferred is placed in close proximity to the coated carrier (the target). ${ }^{1-4}$ However, in the conventional LIFT process the donor material acts as its own propellant, which leads to its unavoidable damage during the transfer process. A variation in the original LIFT technique employed to overcome this problem utilizes an intermediate sacrificial layer called the dynamic release layer (DRL), which is sandwiched between the carrier and the donor to absorb the incident pulse energy. ${ }^{5}$

A tailor-made UV absorbing triazene polymer (TP) has recently gained much favor as a DRL material due to its attractive properties such as low ablation threshold and photolytic dissociation. Upon dissociation, the TP releases $\mathrm{N}_{2}$ gas along with other molecular gaseous fragments, which provide the required push for forward transfer of the overlying donor layer. ${ }^{6}$ Living biological cells, ${ }^{7}$ organic light emitting diodes (OLEDs), ${ }^{8}$ quantum dots, ${ }^{9}$ and ceramic materials ${ }^{10}$ have been transferred using TP as the DRL. ${ }^{11}$ In all these cases the receiver was placed either in contact or in close proximity to the target. However, for an ease in mechanical handling in industrial applications such as those involving transfer of donors for building stacks of different materials, the donor and receiver need to be separated by a relatively large distance. Time-resolved techniques can be used to study the dynamics of the LIFT process for such cases and the quality of the ablated donor material during

\footnotetext{
${ }^{a)}$ Author to whom correspondence should be addressed. Electronic mail: kak@orc.soton.ac.uk.
}

transfer. ${ }^{12,13}$ These observations help in improving understanding of both the ablation and the transfer mechanisms that are directly related to the end scientific and technological applications.

In this paper, conventional nanosecond-shadowgraphy imaging of the TP-DRL assisted LIFT is reported for the solid phase ceramic materials gadolinium gallium oxide $(\mathrm{Gd}-\mathrm{Ga}-\mathrm{O})$ and ytterbium doped yttrium aluminium oxide (Yb:YAG). The time evolution of the LIFT process and the influence of various operating parameters such as the laser fluence and thicknesses of the donor and DRL materials on the quality of the opaque transferred disc (referred to as the "flyer") were also investigated and the results are discussed.

\section{EXPERIMENTAL}

The samples for the nanosecond-shadowgraphic studies were prepared by depositing films of the donor materials $\mathrm{Gd}-\mathrm{Ga}-\mathrm{O}$ and $\mathrm{Yb}$ :YAG on top of previously TP coated fused silica substrates. The TP was synthesized by the method described in Ref. 14 and spin-coated on the silica substrates from solutions of TP in chlorobenzene and cyclohexanone $(1: 1 \mathrm{w} / \mathrm{w})$. The donor material layer was then deposited on top by pulsed laser deposition (PLD). All the donor films were prepared under room temperature conditions and in an oxygen atmosphere $\left(7 \times 10^{-2}\right.$ mbar for $\mathrm{Gd}-\mathrm{Ga}-\mathrm{O}$ and 2 $\times 10^{-3}$ mbar for $\mathrm{Yb}$ :YAG). Due to the temperature sensitivity of the TP, which decomposes at $250{ }^{\circ} \mathrm{C},{ }^{14} \mathrm{Gd}-\mathrm{Ga}-\mathrm{O}$ and $\mathrm{Yb}$ :YAG were chosen as the donor materials because they can be deposited at room temperature. Several samples with different TP thicknesses $(50,150$, and $350 \mathrm{~nm})$ and different donor thicknesses (200 and $1000 \mathrm{~nm}$ ) were prepared to study the effect of the relative thicknesses of the DRL and donor material on the quality of the ablated flyer.

The pump-probe setup used for the experiments is shown in Fig. 1. A XeCl excimer laser (Compex, Lambda Physik, $\lambda=308 \mathrm{~nm}, \tau=30 \mathrm{~ns}$ ) was used as the pump source. The reason for choosing this laser was the strong absorption of the TP at this wavelength. ${ }^{6}$ The second harmonic of a 


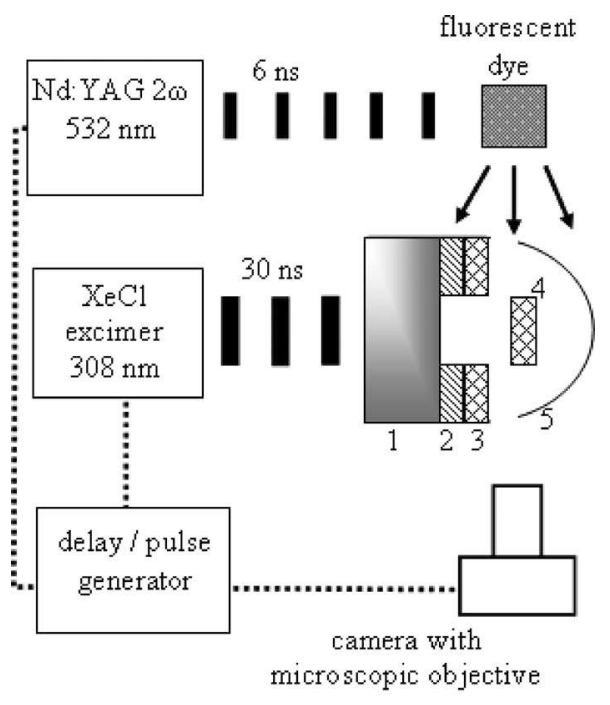

1- Carrier; 2 - TP-DRL;

3 - Donor film; 4- Ejected flyer;

5 - Shockwave front

FIG. 1. Schematic of the nanosecond-shadowgraphy setup.

Nd:YAG laser ( $\lambda=532 \mathrm{~nm}, \tau=6 \mathrm{~ns})$ was used as the probe beam. The pump pulses were centrally incident on a square mask with an aperture of $2 \mathrm{~mm}$, resulting in an approximately homogeneous beam. This uniform beam was then imaged by a lens onto the sample with a demagnification of $4 \times$, yielding a spot size of $500 \times 500 \mu \mathrm{m}^{2}$. The pump laser energy incident on the target was controlled by a variable attenuator plate and was measured by a pyroelectric energy meter (Molectron J4-09 or Gentec QE 50) placed at the end of the beam line. The target was mounted on a motorized translation stage, with the film facing away from the laser beam for back-side ablation.

Visualization of the process was achieved by a complementary metal-oxide semiconductor (CMOS) camera with a microscope objective placed perpendicular to the laser beam (i.e., parallel to the sample surface). The probe beam generated fluorescence from a Rhodamine dye in a quartz cuvette placed on the camera axis on the opposite side of the sample, which in turn illuminated the ejected flyer and the released shockwave. It is well known that an incoherent source is better suited for shadowgraphy than a coherent light source, so the fluorescent dye was used for illumination purposes rather than the direct irradiation from the Nd:YAG laser. ${ }^{15}$ The delay between the pump and the probe beam was controlled using a digital pulse/delay generator (Stanford Research Systems DG535).

\section{RESULTS AND DISCUSSION}

For all the experiments the pump laser was rasterscanned across the sample to selectively ablate an array of square holes with each row corresponding to a different laser fluence value. A computer-controlled system allowed variation in the fluence, exposed position of the sample, the delay time between the pump and probe beam, and capture of a

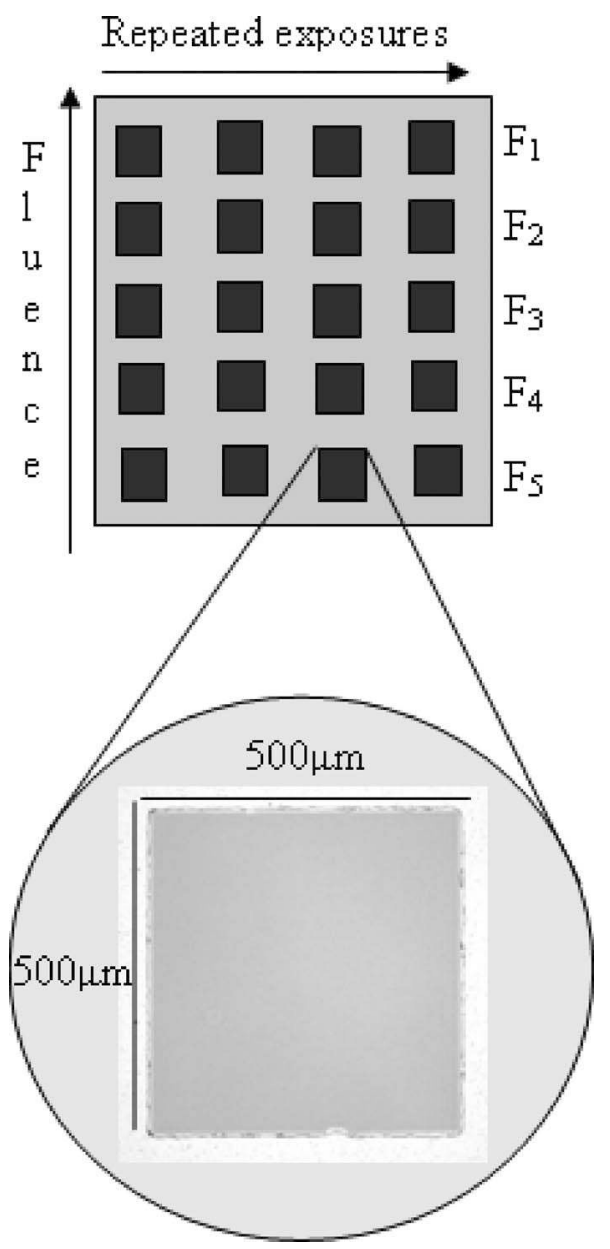

FIG. 2. Schematic of the donor sample ablated using different fluence values (from $F_{1}$ to $F_{5}$ ) for each row (inset shows the optical microscope image of an ablated region on the donor).

sequence of frames showing the generated shockwave and the ejected flyer. Each picture was recorded using a different pulse and corresponded to a different position on the sample. Figure 2 shows the schematic of the ablated matrix generated on the sample, and the inset to the figure shows one of the ablated square patterns $\left(500 \times 500 \mu \mathrm{m}^{2}\right)$ observed under an optical microscope. The results corresponding to the study of the effect of the laser fluence, TP-DRL, and donor thicknesses on the flyer dynamics are discussed below.

\section{A. Laser fluence dependence}

Figure 3 shows time-resolved shadowgraphs of the target having a $1 \mu \mathrm{m}$ thick $\mathrm{Gd}-\mathrm{Ga}-\mathrm{O}$ film deposited on top of a $350 \mathrm{~nm}$ thick TP at fluence values of (a) $\sim 60$ and (b) $\sim 600 \mathrm{~mJ} / \mathrm{cm}^{2}$. The time delay between the pump and the probe pulses was varied from 400 to 2400 ns with a delay step of 400 ns. Figures 4(a) and 4(b) show the images for the second donor material Yb:YAG (1 $\mu \mathrm{m}$ thick) with all other conditions kept the same.

The shockwave released by the huge pressure jump at the ablation site and the opaque flyer ejected from the target surface are clearly visible in all the pictures. It is clear from these images that the higher the fluence, the farther the shockwave and flyer propagate away from the target surface. 


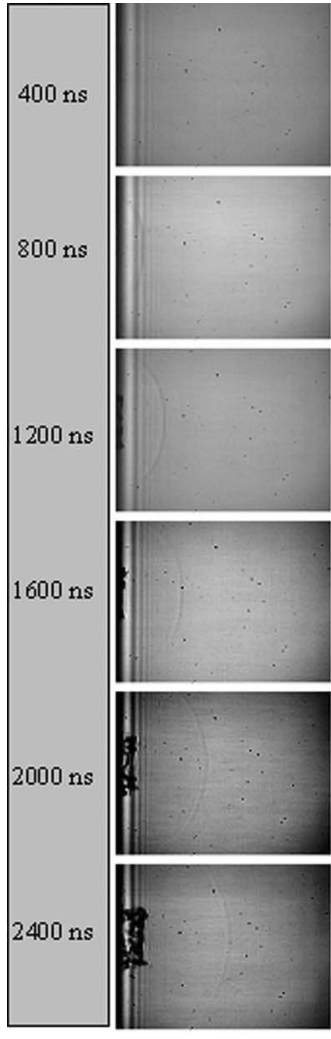

(a)

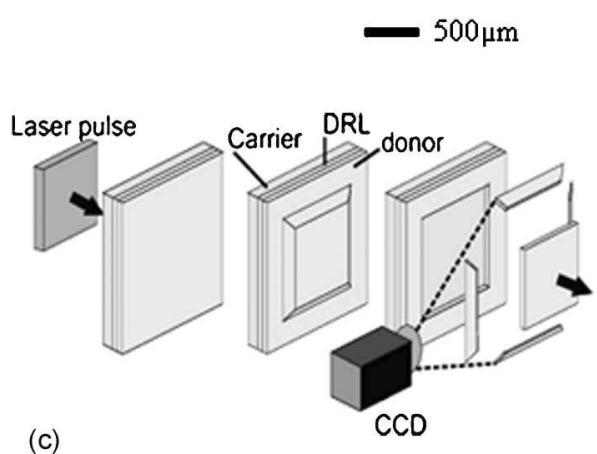

(c)

FIG. 3. Shadowgraphs recorded for $1 \mu \mathrm{m}$ thick Gd-Ga-O target with 350 $\mathrm{nm}$ TP for fluence values of (a) 60 and (b) $600 \mathrm{~mJ} / \mathrm{cm}^{2}$ at delay times of 400-2400 ns between the pump and the probe. The shockwave front and the flyer have been marked as 1 and 2, respectively, as an example in (b). Also the solid chunks of the $\mathrm{Gd}-\mathrm{Ga}-\mathrm{O}$ layer from the shattered edges of its flyer are circled and marked as (a) and (b). The 3D schematic of the donor shearing process is shown in (c).

In Figs. 3(a) and 4(a), for a delay time of $800 \mathrm{ns,} \mathrm{while} \mathrm{the}$ flyer had just started to delaminate from the surface at an irradiance of $60 \mathrm{~mJ} / \mathrm{cm}^{2}$ pulse, it had already moved a substantial distance from the target surface for a $\sim 10 \times$ higher fluence $\left(600 \mathrm{~mJ} / \mathrm{cm}^{2}\right)$ [see Figs. 3(b) and 4(b)]. This can be attributed to the fact that at low fluence the pressure generated by the decomposed polymer was not sufficient to expel the flyer far from the surface. However, as the fluence was increased, the ablation depth also increased, which in turn decomposed a larger fraction of the TP film, and this released more gaseous fragments and hence an increased pressure was exerted on the top donor layer.

Also observed was a distorted shockwave front at the higher fluence value $\left(600 \mathrm{~mJ} / \mathrm{cm}^{2}\right)$ for both the materials.

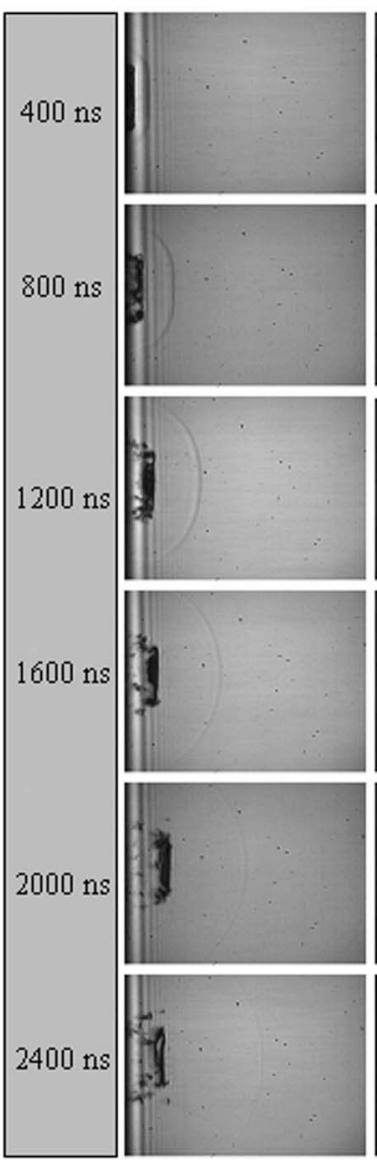

(a)

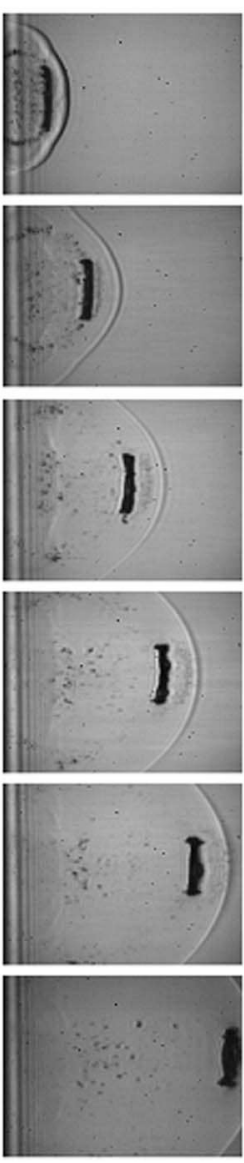

(b)

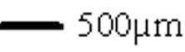

FIG. 4. Images for the $1 \mu \mathrm{m}$ thick Yb:YAG target with $350 \mathrm{~nm}$ TP for fluence values of (a) 60 and (b) $600 \mathrm{~mJ} / \mathrm{cm}^{2}$ at delay times of 400-2400 ns between the pump and the probe.

The reason for this is believed to be the decomposed TP escaping out from the sides of the flyer at the time of ejection due to an extremely high pressure present at the ablation spot, which led to the overlapping side shockwaves. After time delays of around $1600 \mathrm{~ns}$ the shockwave appeared to regain the hemispherical shape [refer to Fig. 3(b)].

On comparing the shadowgraphs of the two ceramic materials it is evident that the Yb:YAG flyer was traveling farther than that of the $\mathrm{Gd}-\mathrm{Ga}-\mathrm{O}$ flyer at all delay times for both fluences. Two additional much smaller flyers were also observed in the case of the $\mathrm{Gd}-\mathrm{Ga}-\mathrm{O}$ donor layer [circled and marked as (a) and (b) in Fig. 3(b)]. These observations can be attributed to the difference in the quality of the two donor films as analyzed by a scanning electron microscope. The Yb:YAG film was somewhat porous and had substantial surface texturing, while the $\mathrm{Gd}-\mathrm{Ga}-\mathrm{O}$ was a much smoother and flatter film. The solid $\mathrm{Gd}-\mathrm{Ga}-\mathrm{O}$ film is likely to offer more resistance to shearing than the porous Yb:YAG layer, which was relatively easily detached, resulting in a cleaner and less violent shearing process. Comparatively less force was therefore required to forward transfer the Yb:YAG layer and hence the greater distance of travel observed by the flyer from this film for the same fluence. The solid material re- 
sulted from the comparatively violent shearing of the flyer from the nonporous solid $\mathrm{Gd}-\mathrm{Ga}-\mathrm{O}$ film, which in turn may have disrupted the edges of the flyer during its detachment from the donor surface. These shattered edges appear as the two smaller flyers when captured by the camera mounted perpendicular to the target surface as depicted in Fig. 3(c). However only fragments are observed for the case of Yb:YAG due to the relatively less violent shearing process and difference in the surface quality. This clearly explains the lesser amount of debris seen in the case of the flyer from the $\mathrm{Yb}$ :YAG film.

The graphs shown in Figs. 5(a) and 5(b) show the distance propagated by the shockwave and the flyer, respectively, as a function of delay time for a range of pump laser fluence values for the $1 \mu \mathrm{m}$ thick Yb:YAG on top of $350 \mathrm{~nm}$ TP-DRL target sample. The solid lines in Figs. 5(a) and 5(b) are simple guides to the eye. The experimental data for the shockwave propagation follow the similar qualitative trend [shown in Fig. 5(c)] as predicted by the theoretical model ${ }^{16,17}$ which is based on Eq. (1),

$$
R=\left\{\left[1.5 C_{5}\left(E_{0}\right)^{1 / 2} t+C_{4}^{3 / 2}\right]^{2 / 3}-C_{4}\right\} / C_{5},
$$

where

$$
\begin{aligned}
& C_{4}=\frac{A \delta}{8}\left(\frac{2}{\gamma+1}\right)^{2} \rho_{s}, \\
& C_{5}=\frac{\rho_{0} A}{\gamma+1}\left(\frac{1}{\gamma-1}+\frac{4}{\gamma+1}\right) .
\end{aligned}
$$

Here $R$ is the propagation distance, $E_{0}$ is the laser energy, $\gamma$ is the specific heat ratio of the atmosphere, $A$ is the laser-ablated area, $\rho_{s}$ is the TP density, $\delta$ is the ablation depth, $\rho_{0}$ is the atmospheric density, and $t$ is the delay time. However, quantitatively the experimental and theoretical values differ by $\sim 60 \%$ as shown in Fig. 5(c). The reason for this discrepancy is the overlying donor layer in the present case, while the theoretical model takes the TP layer into account only. The generated shockwave has to travel through the donor layer, which results in the lower values of distance propagated.

For the case of the flyer the range of incident energy densities spans more than one order of magnitude, and while a straight line fit appears to be appropriate for the lowest incident energy density results $\left(60 \mathrm{~mJ} / \mathrm{cm}^{2}\right)$, the data points for the $680 \mathrm{~mJ} / \mathrm{cm}^{2}$ are seen to depart from a simple straight line fit, as shown in Fig. 5(b). Given that the flyer will experience a frictional damping force from the surrounding air, it is to be expected that some slowing down will occur, particularly for the higher velocities recorded, as the drag experienced by an object is proportional to (velocity) ${ }^{2}$. For this reason, the fits (which remain as guides to the eye) are not shown as straight lines.

The variation in the propagation velocity for both the shockwave and the flyer with the fluence at a delay time of $800 \mathrm{~ns}$ is also shown in Figs. 6(a) and 6(b), respectively, for a sample with $1 \mu \mathrm{m}$ thick $\mathrm{Gd}-\mathrm{Ga}-\mathrm{O}$ donor film on top of a $350 \mathrm{~nm}$ TP-DRL. The velocities for both the shockwave and the flyer increase monotonically with the laser fluence. The
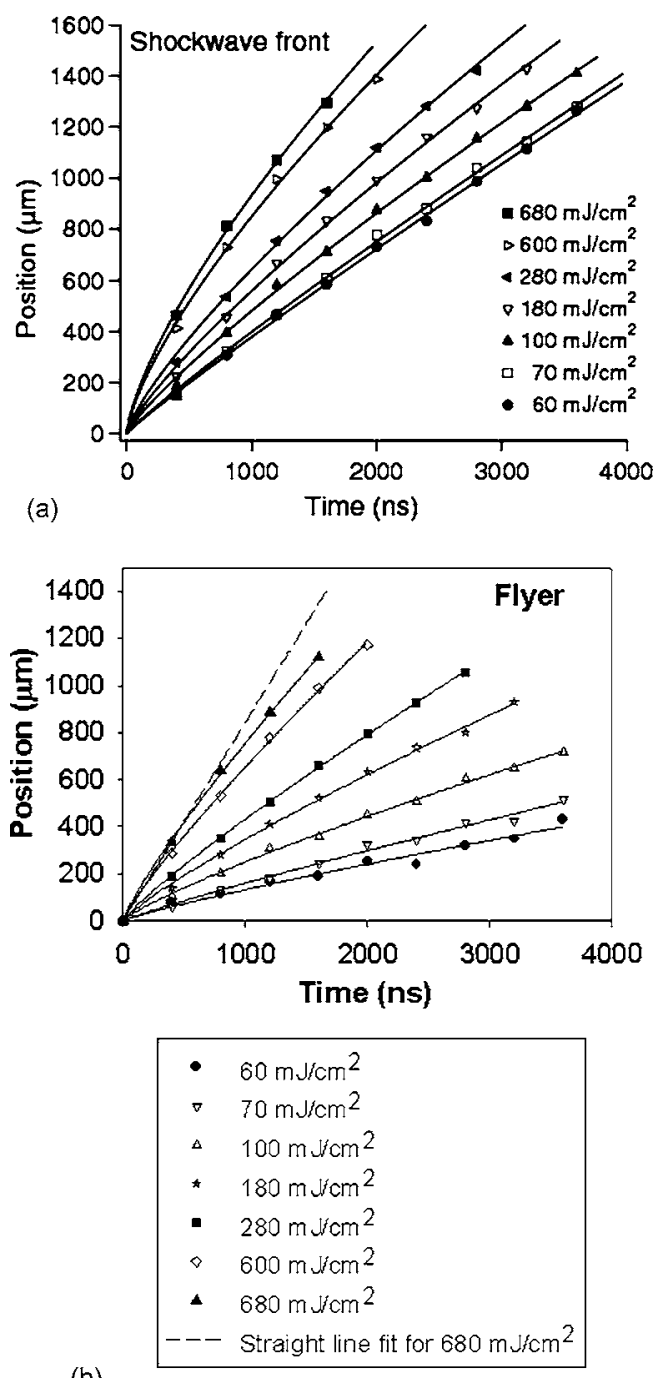

(b)

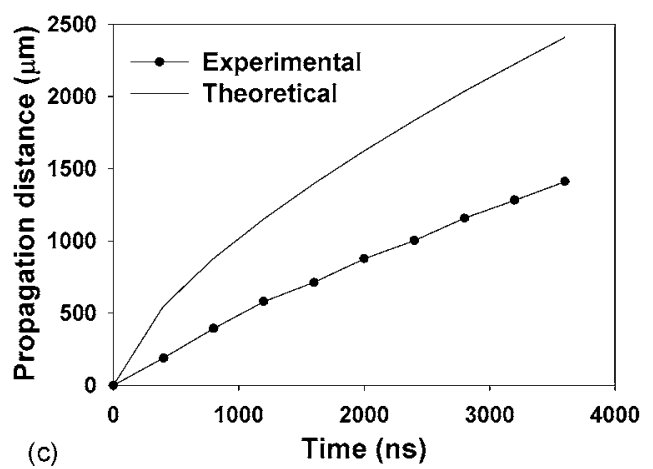

FIG. 5. Variation in the position of the (a) shockwave and the (b) flyer as functions of the pump fluence at different delay times for the sample with $1 \mu \mathrm{m}$ thick Yb:YAG on top of a $350 \mathrm{~nm}$ TP. The flyer is shown to slow down and depart from a constant velocity straight line fit for a fluence of $680 \mathrm{~mJ} / \mathrm{cm}^{2}$ in (b). (c) Plot showing the variation in propagation distance of shockwave as a function of delay time as predicted by the theory and that given by experimental values for $1 \mu \mathrm{m}$ thick Yb:YAG on top of a $350 \mathrm{~nm}$ TP with fluence of $100 \mathrm{~mJ} / \mathrm{cm}^{2}$.

solid line drawn through the experimental data points for the shockwave is just a guide to the eye [Fig. 6(a)]. However the dashed curve drawn along the flyer data points in the log-log plot in Fig. 6(b) is a theoretical fit based on the following model.

The energy density of the incident laser pulse was used to decompose the TP-DRL layer into gaseous fragments, 

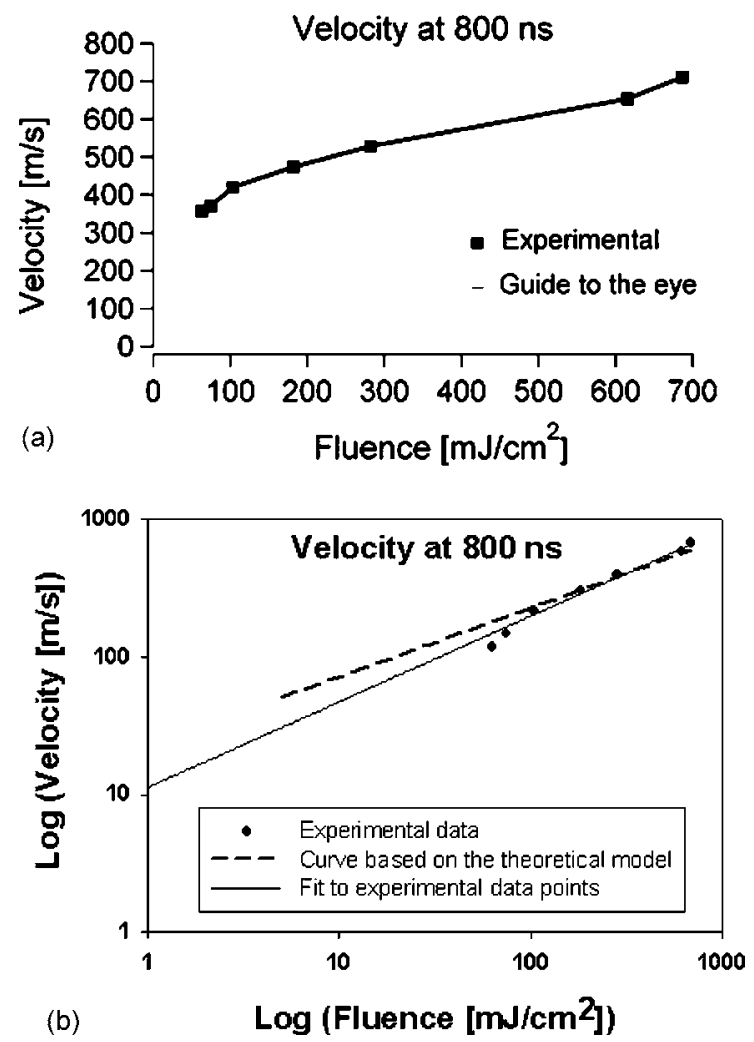

FIG. 6. Variation in the propagation velocity of the (a) shockwave and the (b) flyer as functions of the pump fluence at a delay time of $800 \mathrm{~ns}$ for a sample with $1 \mu \mathrm{m}$ thick Gd-Ga-O donor film on top of a $350 \mathrm{~nm}$ TP-DRL.

which in turn pushed the irradiated donor film forward. Assuming that the incident absorbed energy of the laser pulse was proportional to the kinetic energy of the ejected flyer, i.e.,

$$
E_{\text {pulse }} \propto \frac{1}{2} m v^{2},
$$

where $E_{\text {pulse }}, m$, and $v$ are energy of the laser pulse, mass, and velocity of the ejected flyer, respectively. For fixed values of $m$

$$
\begin{aligned}
& E_{\text {pulse }} \propto v^{2}, \\
& \therefore v=K \sqrt{E_{\text {pulse }}}
\end{aligned}
$$

where $K$ is the proportionality constant. Equation (3) represents the analytical dashed plot of Fig. 6(b), and it is consistent with the experimental data. However it should be mentioned that the experimental data points corresponding to low fluences depart from the theoretical straight line fit based on the above model. This is due to the fact that at these fluence values the laser energy is not sufficient to completely decompose the TP layer; hence a lesser forward push acts on the flyer, leading to a lower value of the flyer velocity. ${ }^{18,19} \mathrm{~A}$ similar trend in the variation in propagation distance and velocity with laser fluence was observed for all the other samples irrespective of the TP-DRL and donor material ( $\mathrm{Gd}-$ $\mathrm{Ga}-\mathrm{O}$ or $\mathrm{Yb}: \mathrm{YAG})$ and their thicknesses.

Based on the previous analysis, we now consider the overall energy budget for the entire LIFT process to better understand the relative pathways for conversion of the incident absorbed laser energy into the kinetic energy of the flyer, the shockwave, and the physical processes behind the DRL decomposition and rupture of the donor film. The efficiency of energy conversion from incident pulse energy to kinetic energy of the flyer was directly inferred from the fit to the experimental data points as shown in Fig. 6(b) for a $1 \mu \mathrm{m}$ thick Gd-Ga-O donor film on top of a $350 \mathrm{~nm}$ TPDRL at a fluence of $600 \mathrm{~mJ} / \mathrm{cm}^{2}$. The kinetic energy of the flyer resulted in $\sim 20 \%$ of the pulse energy using the slope $(\sim 0.62)$ and the $y$-intercept $(\sim 11.2)$ of the fit.

The TP decomposition process is exothermic and the amount of energy released is $6.97 \times 10^{5} \mathrm{~J} / \mathrm{kg}$. The thermal energy released by the decomposition of a $500 \times 500 \mu \mathrm{m}^{2}$ region of a $350 \mathrm{~nm}$ thick TP film is therefore $\sim 68 \mu \mathrm{J}$ (density of triazene $=1.12 \times 10^{3} \mathrm{~kg} / \mathrm{m}^{3}$ ). This is only $4 \%$ of the incident laser energy (for a fluence of $600 \mathrm{~mJ} / \mathrm{cm}^{2}$ ), so the energy released during TP decomposition can be considered to contribute little to the energy balance equation.

What remains therefore is the energy required to rupture or shear the donor film and the residual energy contained within the multiple shockwaves generated. However it should be added that functional devices such as OLEDs ${ }^{8}$ and viable biomaterials ${ }^{7}$ have been transferred using triazene as a DRL and no thermal damage to the printed features was observed/reported.

Apart from the laser fluence, the thickness of the donor (ceramic material) and the underlying TP layer should also play an important role in determining the dynamics of the flyer. For example, if the ablation depth exceeded the TP layer thickness, then the donor would be damaged and the basic idea of achieving an intact transfer using a DRL is defeated. Also for a thicker donor film the flyer would travel a smaller distance as compared to the flyer from a sample with a thinner donor film due to the greater force required to shear a thicker film. The study of the effect of these parameters is discussed in the next two subsections.

\section{B. Donor thickness dependence}

The influence of the donor thickness on the quality of the ejected flyer was studied by performing pump-probe experiments with the delay time between the pump and the probe pulses set at a constant value of $1600 \mathrm{~ns}$. Pump pulses with a constant energy density were raster-scanned across the sample, and the shadowgraphs of the ejected flyer and the shockwave were recorded. For the case of a constant donor thickness the distance propagated by the shockwave or the flyer as a function of the position on the donor should also be constant, provided the DRL film had a uniform thickness. In the present case, a very homogeneous layer of TP-DRL on the silica substrate was obtained by spin-coating. However, the donor layer deposited by PLD had a radial thickness variation. Any deviation from the straight line behavior can thus be attributed to the thickness variation in the donor film and not the TP. Figure 7(a) shows such a plot for a $\mathrm{Gd}-\mathrm{Ga}-\mathrm{O}$ sample having a maximum thickness $(\sim 1.7 \mu \mathrm{m})$ at the center and a minimum thickness $(\sim 1 \mu \mathrm{m})$ around the edges, and Fig. 7(b) shows the schematic of the target with varying donor thickness. The TP thickness was $350 \mathrm{~nm}$ and the fluence was $\sim 100 \mathrm{~mJ} / \mathrm{cm}^{2}$. The layer thicknesses were mea- 

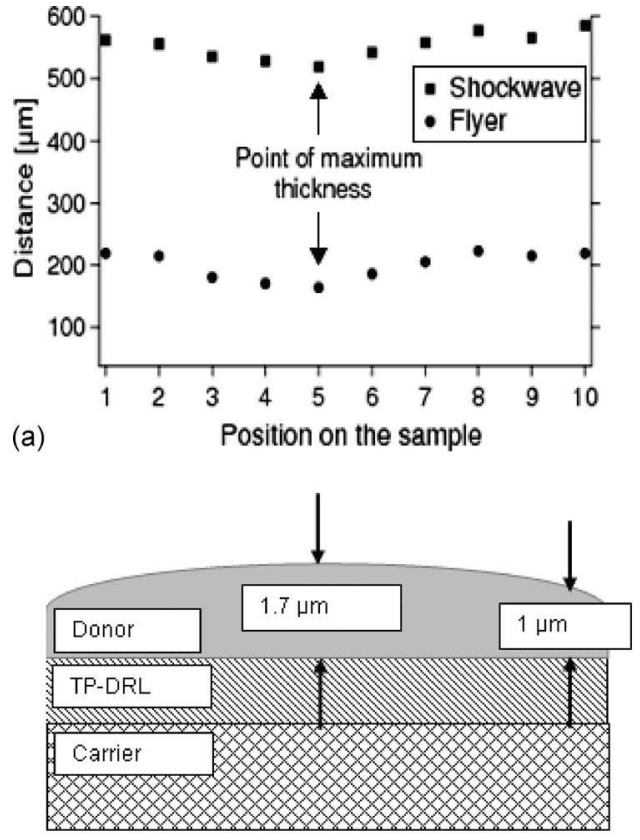

(b)

FIG. 7. (a) Plot showing the dependence of the position of the shockwave and the flyer on the donor thickness for fixed values of TP thickness (350 $\mathrm{nm})$ and delay time (1600 ns) for a $\mathrm{Gd}-\mathrm{Ga}-\mathrm{O}$ sample having a maximum thickness $(\sim 1.7 \mu \mathrm{m})$ at the center and a minimum thickness $(\sim 1 \mu \mathrm{m})$ around the edges. The $x$-axis corresponds to the position on the sample with varying thickness as shown in (b).

sured using a stylus profiler. The dip at the center of the plot (arrowed) coincides with the point of maximum thickness on the target, which suggests a clear dependence of the transfer mechanism on the donor thickness. The graph depicting the decrement of the distance traveled by the flyer with the donor thickness, for the above discussed sample, is shown in Fig. 8. The dashed line represents the analytical fit to the experimental data points. It was obtained using the following simple model.

As mentioned in the previous section the energy of the laser pulse is proportional the kinetic energy of the flyer [Eq. (2)], so for different donor film thicknesses $X_{1}$ and $X_{2}$,

$$
E_{\text {pulse }} \propto \frac{1}{2} m_{1} v_{1}^{2}=\frac{1}{2} m_{2} v_{2}^{2},
$$

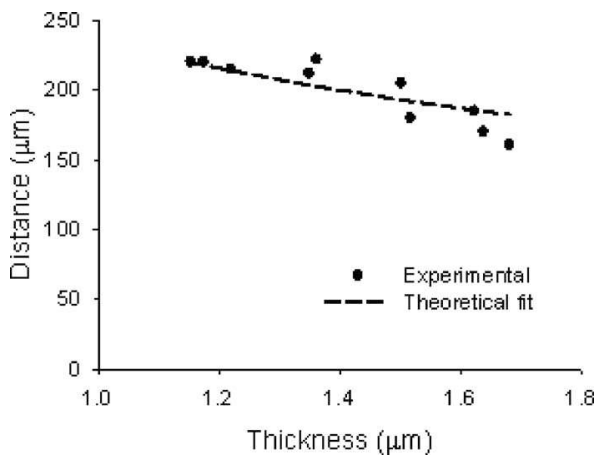

FIG. 8. The propagation distance of the flyer plotted against the thickness of the donor film of $\mathrm{Gd}-\mathrm{Ga}-\mathrm{O}$ for fixed values of TP $(350 \mathrm{~nm})$ and delay time (1600 ns). The curve clearly shows that thicker films have slower velocities, as expected.

$$
\therefore \frac{m_{1}}{m_{2}}=\frac{v_{2}^{2}}{v_{1}^{2}} \text {, }
$$

where $m_{1}$ and $m_{2}$ are the masses of the ejected flyer corresponding to the thicknesses $X_{1}$ and $X_{2}$, respectively, and $v_{1}$ and $v_{2}$ are the corresponding velocities. For a given distance $d$ traveled by the flyer and at a given time $t$,

$$
\begin{aligned}
& d=v t, \\
& \therefore v^{2}=\frac{d^{2}}{t^{2}}, \\
& \Rightarrow v^{2} \propto d^{2} .
\end{aligned}
$$

From Eqs. (4) and (5) we obtain

$$
\begin{aligned}
& \therefore \frac{m_{1}}{m_{2}}=\frac{d_{2}^{2}}{d_{1}^{2}}, \\
& \Rightarrow \frac{d_{2}}{d_{1}}=\sqrt{\frac{m_{1}}{m_{2}}}=\sqrt{\frac{X_{1}}{X_{2}}}, \\
& \therefore d_{2}=d_{1} \sqrt{\frac{X_{1}}{X_{2}}} .
\end{aligned}
$$

Here $d_{1}$ and $d_{2}$ represent the distances traveled by the flyer corresponding to thicknesses $X_{1}$ and $X_{2}$, respectively. Equation (6) represents the theoretical curve plotted in Fig. 8 and is consistent with the experimental data.

\section{TP thickness dependence}

To examine the effect of the DRL layer thickness on the transfer process, shadowgraphy experiments were performed for target samples with two different thickness values of TP (50 and $350 \mathrm{~nm}$ ) while keeping the central thickness of the donor material approximately constant (1 $\mu \mathrm{m}$ Yb:YAG). Figures 9(a) and 9(b) show the recorded time-resolved images of the $\mathrm{Yb}$ :YAG flyers with pulses of $\sim 100 \mathrm{~mJ} / \mathrm{cm}^{2}$ and at delay times of 0-3600 ns between the pump and the probe for these two thickness values of TP. Although we have only studied two different thicknesses of TP, we present the key observations and our qualitative conclusions from these shadowgraphs, which are stated below.

(a) It appears that the delamination of the donor material started earlier for samples with a thicker TP layer (350 $\mathrm{nm}$ ) as compared to those with thinner TP layer (50 $\mathrm{nm})$. For example, even after $400 \mathrm{~ns}$ only a weak shockwave was visible for the sample with a $50 \mathrm{~nm}$ TP-DRL, while the flyer and shockwave were both clearly visible for the $350 \mathrm{~nm}$ TP sample.

(b) The ejected flyer traveled a significantly greater distance for the sample with the thicker TP-DRL film (350 $\mathrm{nm})$ than for the one with the thinner TP layer $(50 \mathrm{~nm})$. For example, after $1600 \mathrm{~ns}$ the flyer from the former sample was already hundreds of microns away from the donor surface, while the flyer from the latter target was barely detached from the surface of the donor. 

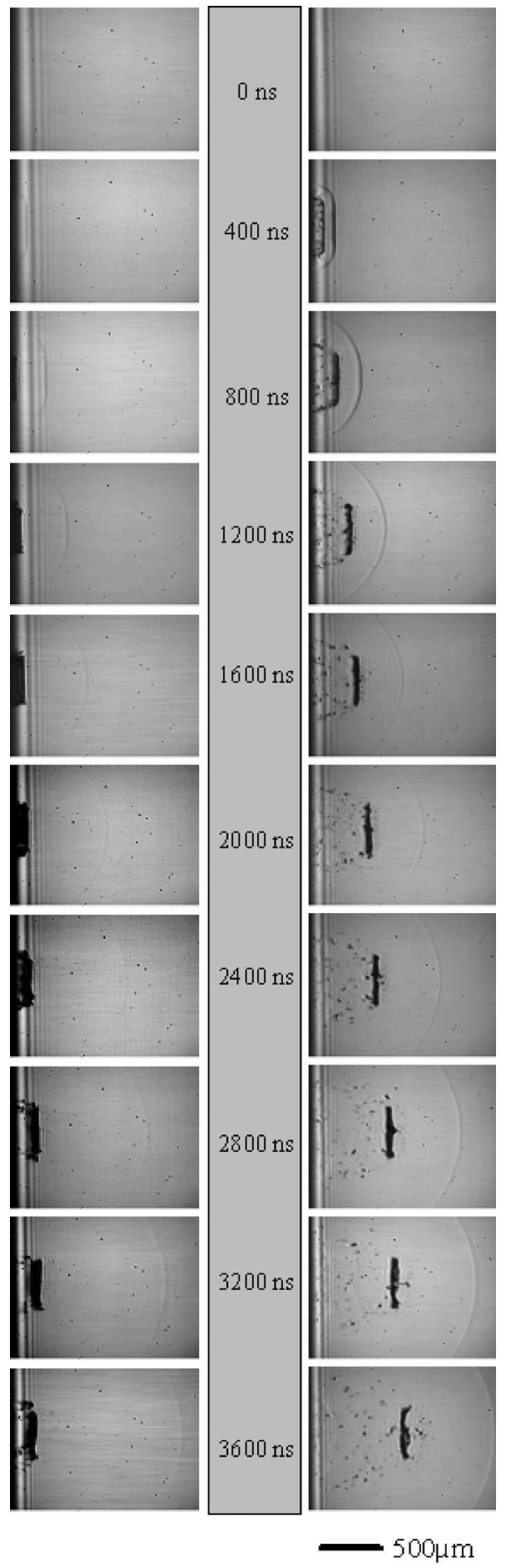

\section{(a) $50 \mathrm{~nm} \mathrm{TP}$}

(b) $350 \mathrm{~nm} \mathrm{TP}$

FIG. 9. Shadowgraphs recorded for targets with (a) 50 and (b) $350 \mathrm{~nm}$ TP-DRL while keeping the donor (Yb:YAG) thickness constant $(\sim 1 \mu \mathrm{m})$ at the delay times of 0-3600 ns between the pump and the probe and a fluence of $100 \mathrm{~mJ} / \mathrm{cm}^{2}$.

(c) The flyer from the sample with the thicker TP appears to be associated with noticeably more debris than the one from the thinner TP.

(d) The flyer started to disintegrate and deform in shape with increasing distance. This effect was more prominent for targets with thicker TP layers.

The explanation for these observations is based on the decomposition of the DRL layer beneath the donor film into gaseous fragments by the pump pulse, which then exerted a forward push on the donor material. The thicker TP layer applied a greater force on the ejected flyer causing its early delamination and providing it with a greater kinetic energy to propagate farther from the sample surface. This long distance travel of the flyer is useful for any practical industrial applications that require large donor-receiver separations, e.g., for transferring multilayers of dissimilar materials sequentially. In contrast, the lesser pressure exerted on the flyer for the sample with a thinner TP layer explains the smaller distance traveled by the flyer and its delayed delamination. The pump pulse decomposed the TP-DRL layer over the irradiated region, thereby shearing the flyer apart from the donor surface by the exerted pressure. For the thicker TP-DRL sample the huge pressure exerted on the flyer made this shearing a violent process and shattered the edges of the flyer during its detachment from the surface, and this may well explain the debris seen in the images for the thicker TP samples.

It should be mentioned that the ablation depth depends not only on the fluence but on TP-DRL thickness as well. ${ }^{18}$ For a given fluence the absolute amount of ablated TP increases with increasing film thickness, thereby providing more thrust for flyer ejection with thicker DRLs; however beyond a certain value of thickness (beyond the ablation depth), the relative percentage of undecomposed TP increases. ${ }^{19}$ For example, at $100 \mathrm{~mJ} / \mathrm{cm}^{2}$ a $50 \mathrm{~nm}$ TP film is completely dissociated, while $\sim 30 \%$ of the TP remains undecomposed for the sample with a $350 \mathrm{~nm}$ TP-DRL layer (data interpolated from that presented in Ref. 18). So the flyer from this sample was bilayered, i.e., consists of both the ceramic material (Yb:YAG or $\mathrm{Gd}-\mathrm{Ga}-\mathrm{O}$ ) and the remaining TP. This undissociated TP could also be a possible cause of the debris seen in the shadowgraphs apart from the shattered edges of the flyer. The actual reason behind this debris is not known for the present case; however this can be very well investigated using an experimental setup including a receiver substrate to collect the flyer along with the debris.

Also the quality of the flyer deteriorated as it moved away from the target surface. Therefore, for an intact long distance transfer of the flyer, an optimal thickness of the sacrificial layer has to be chosen, taking into account the fluence, donor material, and thickness. As stated earlier however, these experiments were only performed for two different TP thicknesses, and hence definitive and fully quantitative conclusions must await a much more systematic study of what is actually a complex and coupled problem.

\section{CONCLUSION}

The dependence of the TP-DRL assisted LIFT process on laser fluence and thickness of the donor and the TP-DRL was studied using a time-resolved nanosecondshadowgraphy technique for solid phase ceramic $\mathrm{Gd}-\mathrm{Ga}-\mathrm{O}$ and $\mathrm{Yb}$ :YAG materials. The propagation distance and velocity for both the shockwave and the flyer were found to increase with the laser fluence. The distance travelled by the ejected flyer decreases with increasing donor film thickness. Finally, the investigation of the effect of the thickness of the 
TP layer on the process revealed that an optimal value of the TP-DRL thickness is required and that the optimal thickness varied with donor material, donor thickness, and the laser energy.

\section{ACKNOWLEDGMENTS}

Financial support from the Engineering and Physical Sciences Research Council (EPSRC), UK (under Grant No. EP/C515668/1), and the Swiss National Science Foundation is gratefully acknowledged.

${ }^{1}$ J. Bohandy, B. F. Kim, and F. J. Adrian, J. Appl. Phys. 60, 1538 (1986). ${ }^{2}$ K. D. Kyrkis, A. A. Andreadaki, D. G. Papazoglou, and I. Zergioti, in Recent Advances in Laser Processing of Materials, edited by J. Perrière, E. Millon, and E. Fogarassy (Elsevier, Amsterdam, 2006).

${ }^{3}$ D. P. Banks, C. Grivas, J. D. Mills, R. W. Eason, and I. Zergioti, Appl. Phys. Lett. 89, 193107 (2006).

${ }^{4}$ I. Zergioti, S. Mailis, N. Vainos, C. Fotakis, S. Chen, and C. Grigoropoulos, Appl. Surf. Sci. 127-129, 601 (1998).

${ }^{5}$ W. A. Tolbert, I.-Y. S. Lee, M. M. Doxtader, E. W. Ellis, and D. D. Dlott, J. Imaging Sci. Technol. 37, 411 (1993).

${ }^{6}$ T. Lippert, Adv. Polym. Sci. 168, 51 (2004)

${ }^{7}$ A. Doraiswamy, R. Narayan, T. Lippert, L. Urech, A. Wokaun, M. Nagel,
B. Hopp, M. Dinescu, R. Modi, R. Auyeung, and D. Chrisey, Appl. Surf. Sci. 252, 4743 (2006).

${ }^{8}$ R. Fardel, M. Nagel, F. Nüesch, T. Lippert, and A. Wokaun, Appl. Phys. Lett. 91, 061103 (2007).

${ }^{9}$ J. Xu, J. Liu, D. Cui, M. Gerhold, A. Y. Wang, M. Nagel, and T. K. Lippert, Nanotechnology 18, 025403 (2007).

${ }^{10}$ D. P. Banks, K. Kaur, R. Gazia, R. Fardel, M. Nagel, T. Lippert, and R. W. Eason, Europhys. Lett. 83, 38003 (2008).

${ }^{11}$ M. Nagel, R. Fardel, P. Feurer, M. Häberli, F. Nüesch, T. Lippert, and A. Wokaun, Appl. Phys. A: Mater. Sci. Process. 92, 781 (2008).

${ }^{12}$ I. Zergioti, A. Karaiskou, D. Papazoglou, C. Fotakis, M. Kapsetaki, and D. Kafetzopoulos, Appl. Surf. Sci. 247, 584 (2005).

${ }^{13}$ M. Hauer, D. J. Funk, T. Lippert, and A. Wokaun, Opt. Lasers Eng. 43, 545 (2005).

${ }^{14}$ M. Nagel, R. Hany, T. Lippert, M. Molberg, F. Nüesch, and D. Rentsch, Macromol. Chem. Phys. 208, 277 (2007).

${ }^{15}$ G. S. Settles, Schlieren and Shadowgraph Techniques: Visualizing Phenomena in Transparent Media (Springer-Verlag, Berlin, 2001).

${ }^{16}$ D. A. Freiwald, J. Appl. Phys. 43, 2224 (1972).

${ }^{17}$ L. S. Bennett, T. Lippert, H. Furutani, H. Fukumura, and H. Masuhara, Appl. Phys. A: Mater. Sci. Process. 63, 327 (1996).

${ }^{18}$ R. Fardel, M. Nagel, T. Lippert, F. Nüesch, A. Wokaun, and B. S. Luk'yanchuk, Appl. Phys. A: Mater. Sci. Process. 90, 661 (2008).

${ }^{19}$ R. Fardel, M. Nagel, F. Nüesch, T. Lippert, and A. Wokaun, Appl. Surf. Sci. 255, 5430 (2009). 